Supporting Information for

\title{
Mechanism-Guided Design and Discovery of Efficient \\ Cytochrome P450-Derived C-H Amination Biocatalysts
}

\author{
Viktoria Steck ${ }^{\ddagger}$, Joshua N. Kolev ${ }^{\ddagger}$, Xinkun Ren, and Rudi Fasan* \\ Department of Chemistry, University of Rochester, 14627 Rochester, New York, USA \\ ‡ Equal contribution. \\ * Correspondence should be addressed to R.F. (rfasan@ur.rochester.edu)
}

Table of contents:

Supplementary Figures S1-S3

Pages S2-S7

Supplementary Tables S1-S3

Pages S8-S11

Supplementary Scheme S1

Page S12

References

Page S13 


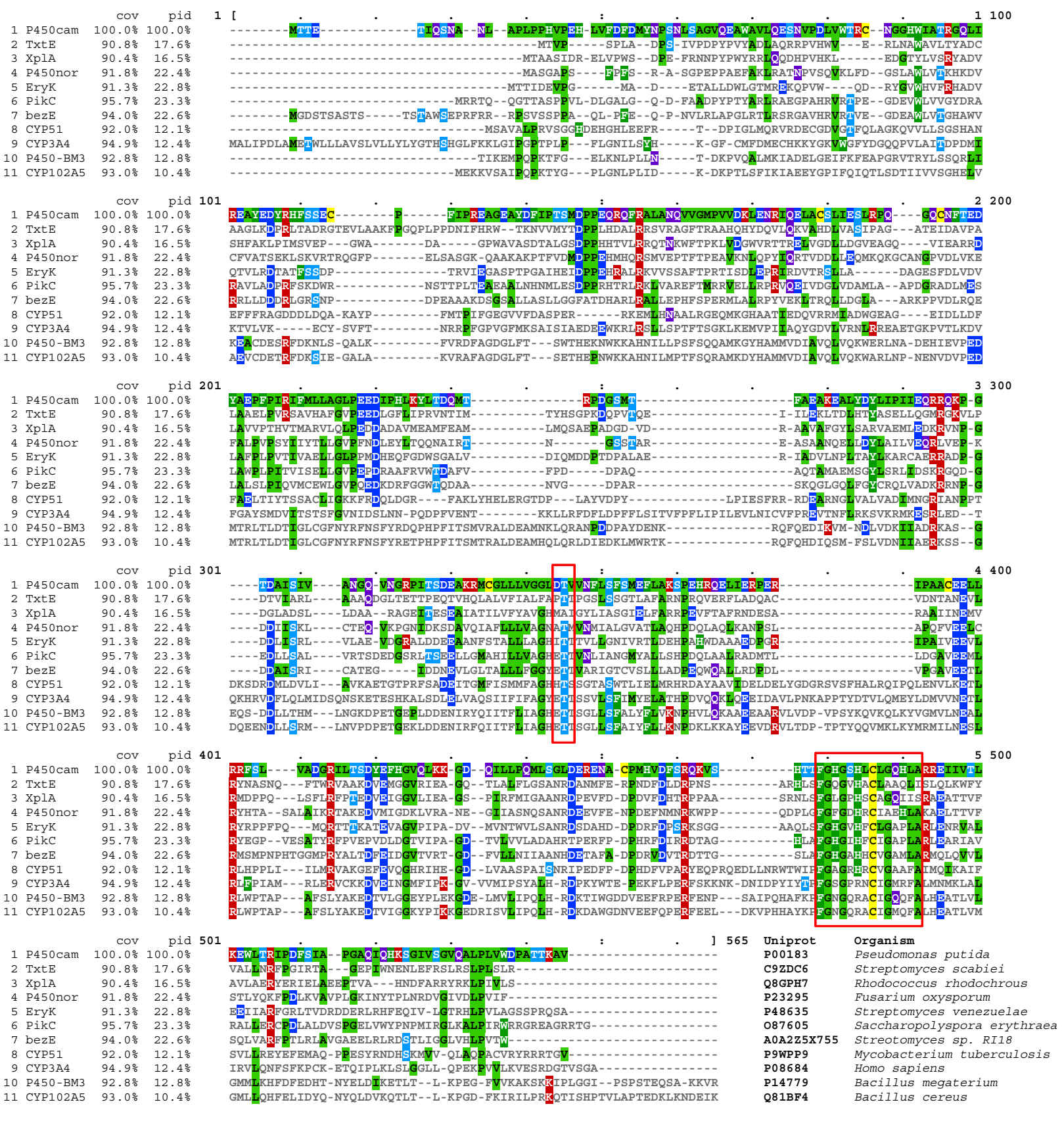

Figure S1. Multiple sequence alignment of P450вм3, XplA, BezE and other cytochromes P450.

The sequence alignment was constructed using the EMBL-EBI tool Clustal Omega ${ }^{1}$ and formatted using MView. ${ }^{2}$ Amino acid residues are color-coded by identity and query coverage (cov) and percent identity (pid) relative to the reference sequence $\mathrm{P} 450_{\mathrm{cam}}$ are indicated. The regions 
encompassing the conserved threonine residue and the heme-binding loop are highlighted with red boxes. Amino acid sequences (Uniprot) used are as follows: P450 cam, P00183, Pseudomonas putida; TxtE, C9ZDC6, Streptomyces scabiei; XplA, Q8GPH7, Rhodococcus rhodochrous; P450nor or CYP55A1, P23295, Fusarium oxysporum; P450 EryK or CYP113A1, P48635, Saccharopolyspora erythraea; P450 PikC or CYP107L1, O87605, Streptomyces venezuelae; bezE, A0A2Z5X755, Streotomyces sp. RI18; CYP51, P9WPP9, Mycobacterium tuberculosis; CYP3A4, P08684, Homo sapiens; P450вм3 or CYP102A1, P14779, Bacillus megaterium; CYP102A5, Q81BF4, Bacillus cereus. 
Figure S2. HPLC traces of a) hemin, b) heme extracted from FL\#62, c) heme extracted from FL\#62 incubated with $\mathrm{Na}_{2} \mathrm{~S}_{2} \mathrm{O}_{4}$, d) heme extracted from FL\#62 reaction with 2,4,6triisopropylbenzenesulfonyl azide 1a, and e) heme extracted from FL\#62(H266F,T268V) reaction with 2,4,6-triisopropylbenzenesulfonyl azide 1a. Reaction conditions: Reactions were conducted as described in the Synthetic Procedures using $2 \mu \mathrm{M}$ P450, with (c-e) or without $10 \mathrm{mM} \mathrm{Na} 2 \mathrm{~S}_{2} \mathrm{O}_{4}$ (a-b), with (d-e) or without $10 \mathrm{mM} \mathrm{1a}(\mathrm{a}-\mathrm{c})$, in $50 \mathrm{mM}$ phosphate buffer ( $\mathrm{pH}$ 7.0). After 10 minutes, the reaction mixture was extracted twice with $0.5 \mathrm{~mL}$ methyl ethyl ketone, and the combined organic layers removed under reduced pressure. The residue was dissolved in $100 \mu \mathrm{L}$ methanol and analyzed by C18 RP-HPLC.

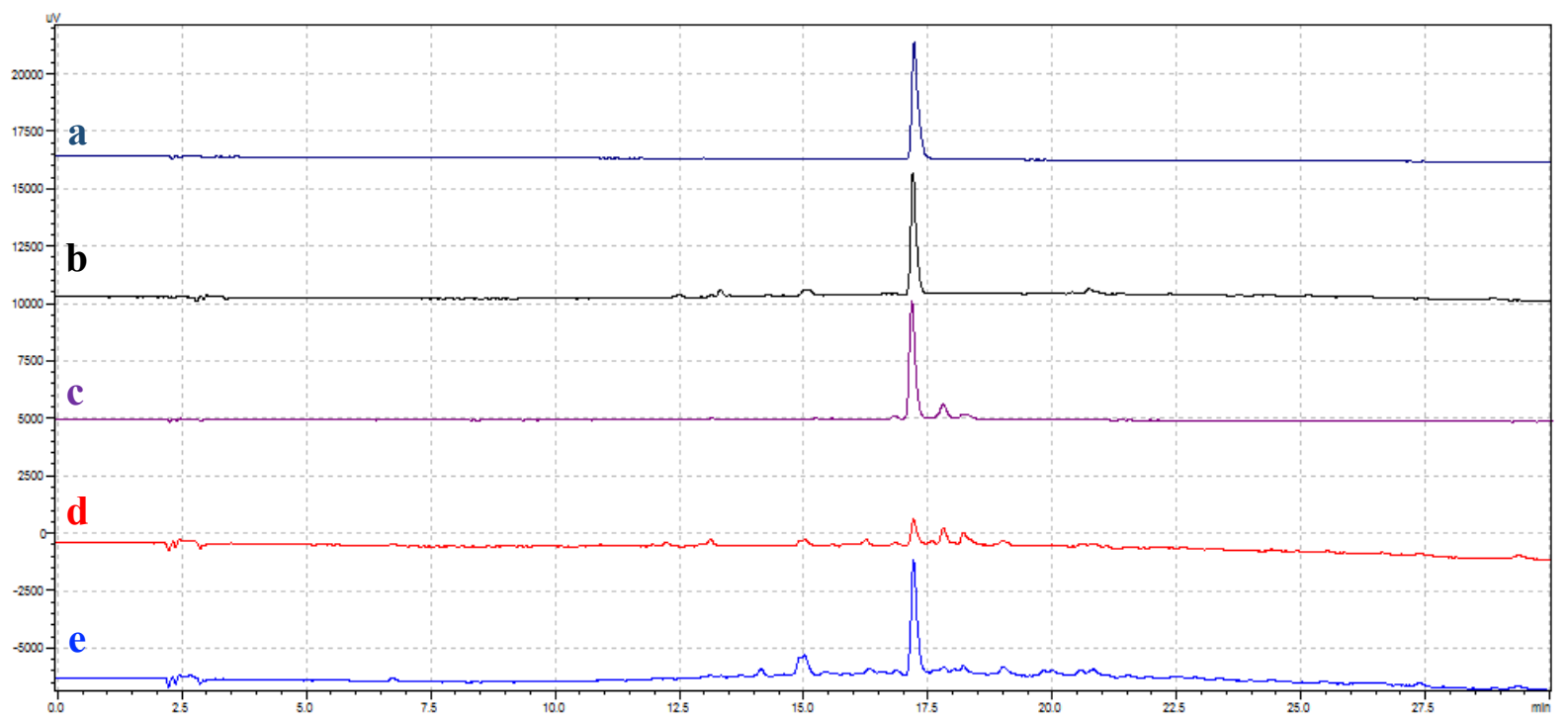


Figure S3. GC-MS traces and EI-MS spectra of products from the intra- and intermolecular H/D competition experiments. KIE values were obtained from integration of the MS signals corresponding to the characteristic fragmentation products with $m / z$ of 227.1 (C- $-\mathrm{H}$ amination) and 226.1 ( $\mathrm{C}-\mathrm{D}$ amination) for the intra-, and $\mathrm{m} / \mathrm{z}$ of 224.1 (non-deuterated) and 229.1 (deuterated) for the intermolecular reaction. The insert boxes highlight the corresponding $\mathrm{m} / \mathrm{z}$ values used for calculation of the KIE values.

a) GC-MS trace and EI-MS spectrum for P450-catalyzed intramolecular H/D competition reaction with $4 \mathbf{a}-d 3$.<smiles>[2H]C(C)c1cc(C([2H])([2H])[2H])c(S(N)(=O)=O)c(C([2H])([2H])C)c1</smiles>

4a- $d 3$

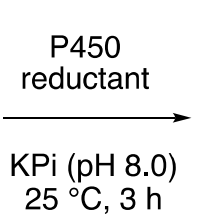

$25^{\circ} \mathrm{C}, 3 \mathrm{~h}$

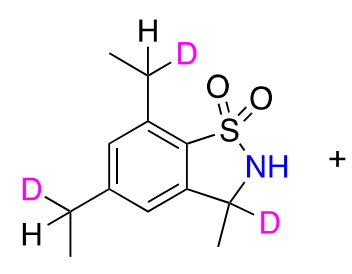

$4 b-d 3$<smiles>[2H]C([2H])([2H])c1cc(C([2H])([2H])C)cc2c1S(=O)(=O)NC2C</smiles>

$4 b-d 2$<smiles>[2H]C(C)c1cc(C([2H])([2H])[2H])c(S(N)(=O)=O)c(C([2H])([2H])[OH2+])c1</smiles>

$4 c-d 3$

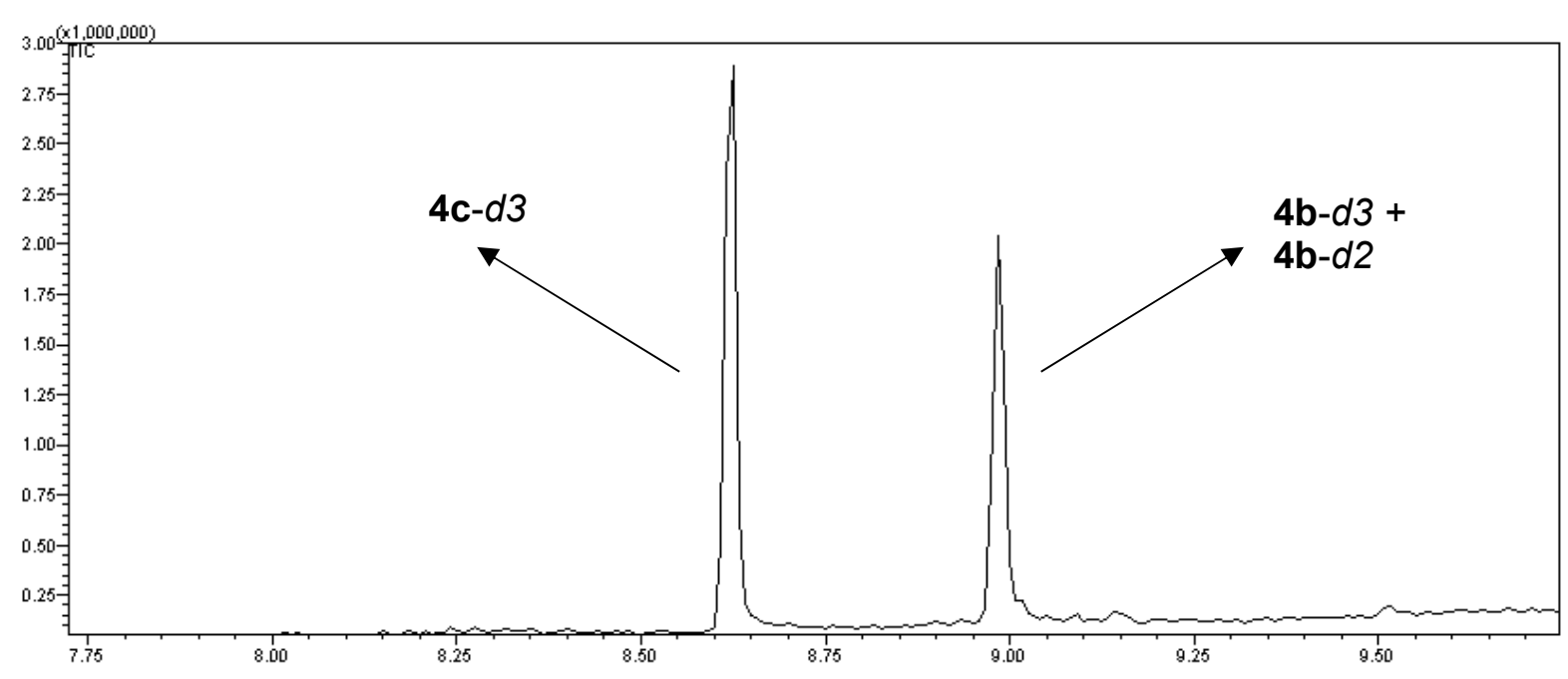


4b- $d 3+\mathbf{4 b}-d 2$ :

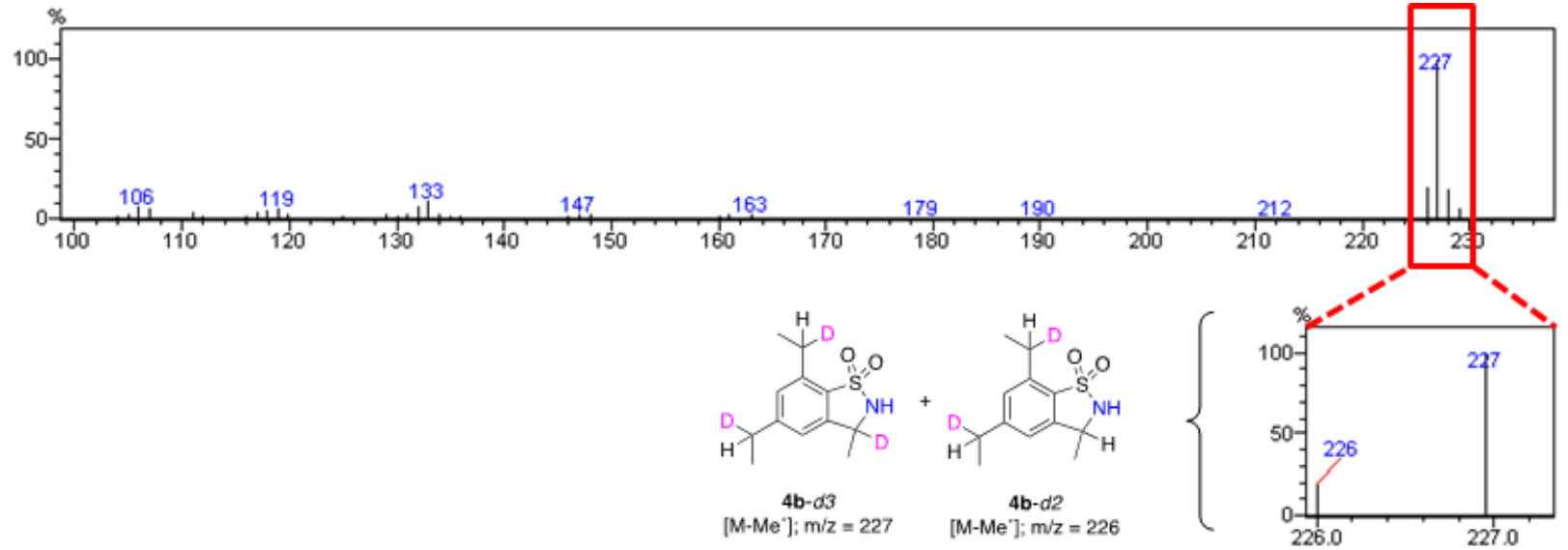

b) GC-MS trace and EI-MS spectra for P450-catalyzed intermolecular H/D competition reaction with $\mathbf{4 a}$ and $\mathbf{4 a}-d 6$.<smiles>CCc1cc(C(C)C)c(S(N)(=O)=O)c(C(C)C)c1</smiles>

4a<smiles>[2H]C([2H])([2H])c1cc(C([2H])([2H])[2H])c([N+](=O)[O-])c(C([2H])(C)C)c1</smiles>

4a- $d 6$

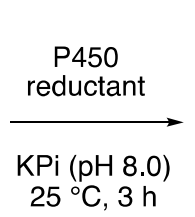

$25^{\circ} \mathrm{C}, 3 \mathrm{~h}$<smiles>CC(C)c1cc(C(C)C)c2c(c1)[C@H](C)NS2(=O)=O</smiles>

$4 b$<smiles>[2H]C([2H])([2H])c1cc(C([2H])(C)C)c2c(c1)C([2H])(C)NS2(=O)=O</smiles>

$4 \mathbf{b}-d 5$<smiles>[2H]C([2H])([2H])c1cc(C([2H])([2H])C)cc(C([2H])(C)C)c1S(N)(=O)=O</smiles>

$4 c$

4c- $d 6$

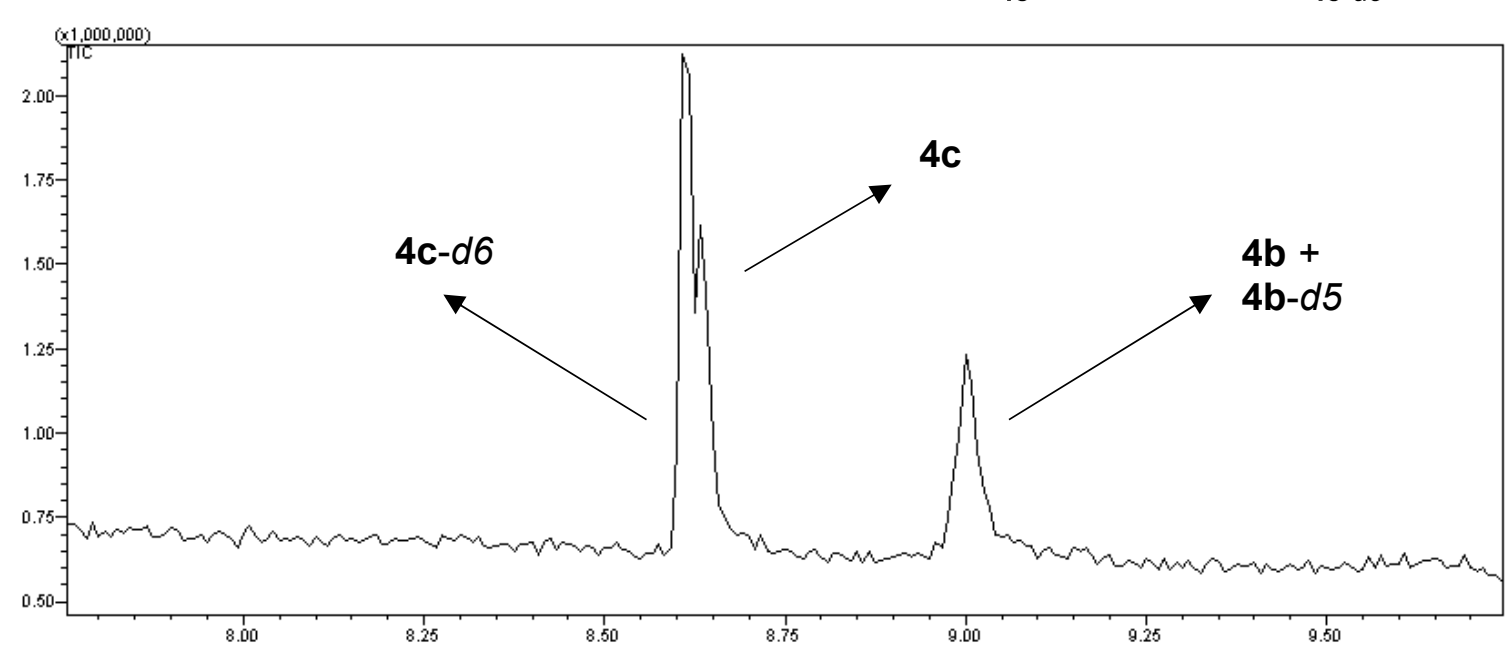




\section{4c- $d 6$ :}

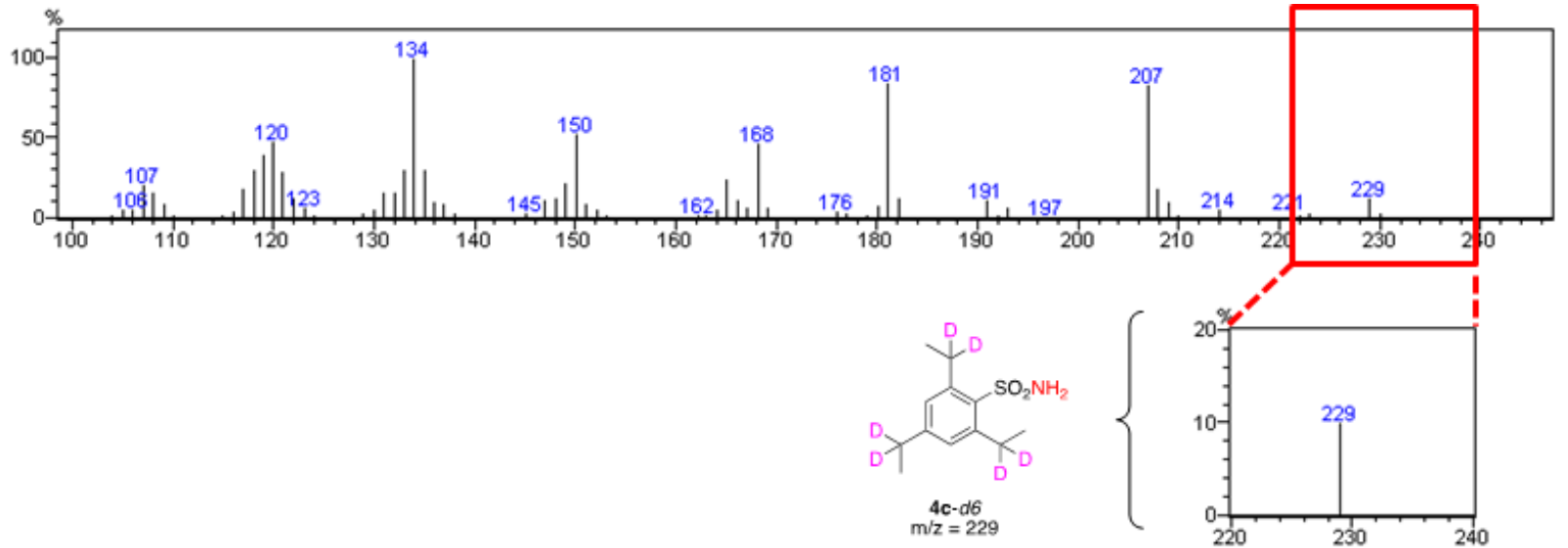

4c:

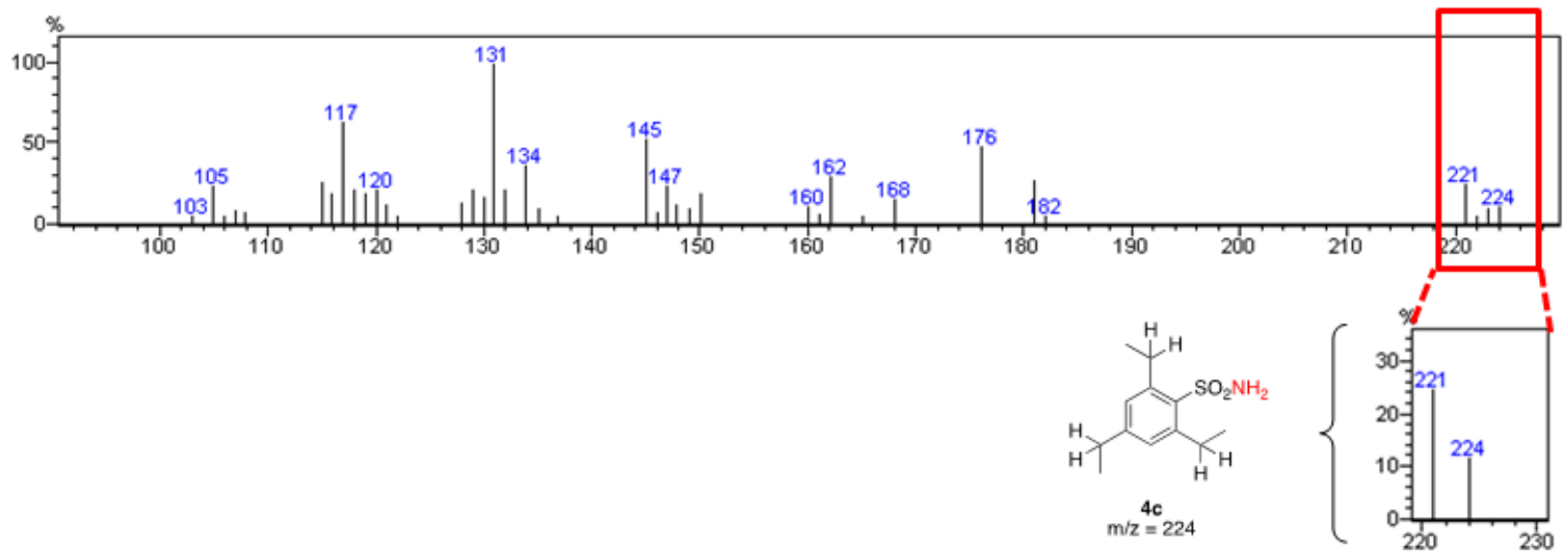

4b- $d 3+4 \mathbf{b}-d 2$ :

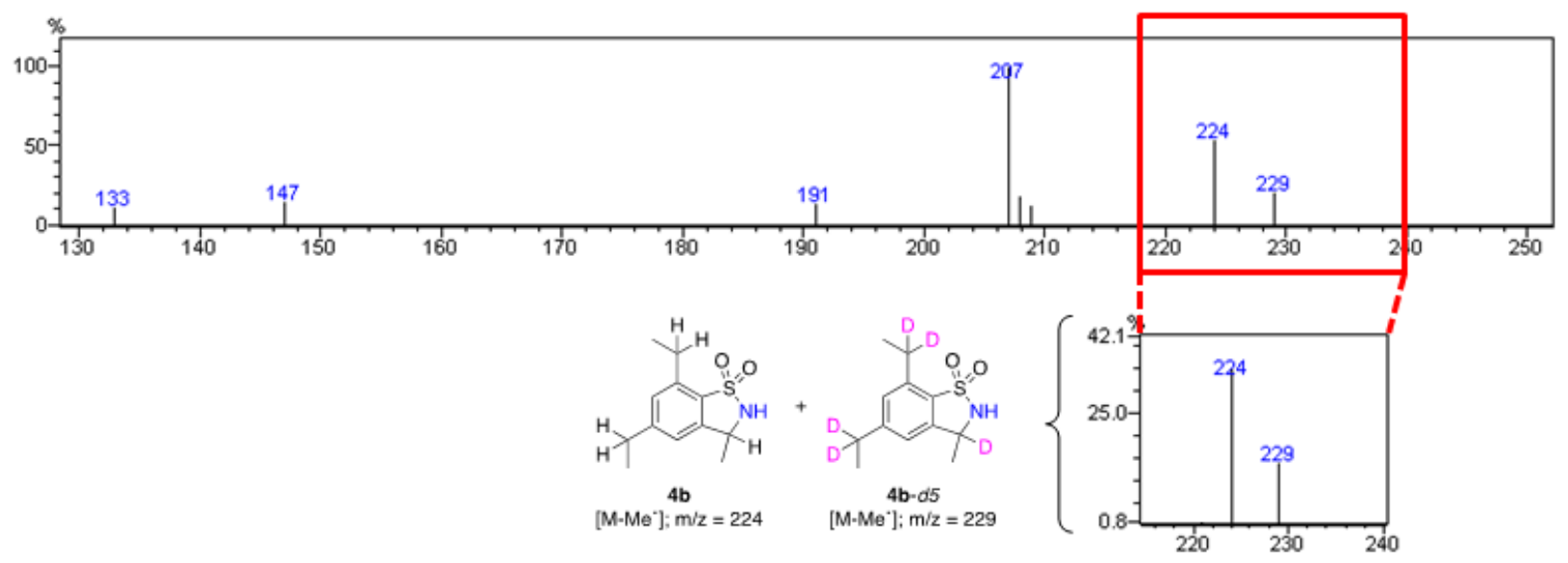


Table S1. Catalytic amination activity and selectivity of engineered $\mathrm{P}^{4} 50_{\mathrm{BM} 3}$ variants toward the $\mathrm{C}-\mathrm{H}$ amination of 2,4,6-triethylbenzenesulfonyl azide $\mathbf{4 a}$. $^{\mathrm{a}}$<smiles>CCc1cc(CC)c(S(N)(=O)=O)c(CC)c1</smiles>

$4 a$

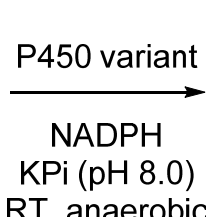

$\mathrm{RT}$, anaerobic

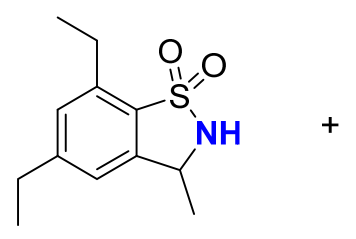

4b<smiles>CCc1cc(CC)c(S(N)(=O)=O)c(CC)c1</smiles>

4c

\begin{tabular}{cccc}
\hline Entry & Variant & TTN $^{\mathrm{b}}$ & Selectivity (\%) $^{\mathrm{c}}$ \\
\hline 1 & FL\#62 & $34 \pm 4$ & $17 \%$ \\
2 & FL\#62(H266F) & $56 \pm 5$ & $21 \%$ \\
3 & FL\#62(E267A) & $24 \pm 4$ & $8 \%$ \\
4 & FL\#62(T268A) & $110 \pm 5$ & $21 \%$ \\
5 & FL\#62(T268V) & $110 \pm 15$ & $28 \%$ \\
6 & FL\#62(T438V) & $63 \pm 20$ & $15 \%$ \\
7 & FL\#62(H266F,T268V) & $170 \pm 25$ & $28 \%$ \\
8 & FL\#62(E267A,T268V) & $150 \pm 10$ & $23 \%$ \\
9 & FL\#62(E267A,T438V) & $39 \pm 12$ & $11 \%$ \\
10 & FL\#62(T268V,T438V) & $52 \pm 5$ & $29 \%$ \\
11 & FL\#62(H266F,T268V,T438V) & $170 \pm 15$ & $31 \%$ \\
12 & FL\#62(H266F,T268V,P392G) & $170 \pm 25$ & $14 \%$ \\
13 & FL\#62(H266F,T268V,F393P) & $130 \pm 10$ & $10 \%$ \\
14 & FL\#62(H266F,T268V,G394P) & $230 \pm 25$ & $9 \%$ \\
15 & FL\#62(H266F,T268V,I401P) & $170 \pm 12$ & $26 \%$ \\
16 & FL\#62(H266F,T268V,G402P) & $210 \pm 15$ & $10 \%$ \\
\hline 0.5 & & & \\
\hline
\end{tabular}

a $0.5 \mu \mathrm{M}$ P450, $3 \mathrm{mM}$ substrate, $10 \mathrm{mM}$ NADPH, $50 \mathrm{mM} \mathrm{KPi} \mathrm{(pH} \mathrm{8.0),} \mathrm{room} \mathrm{temperature,}$ anaerobic conditions.

$\mathrm{b}$ Total turnover numbers ( $\mathrm{mol}$ sultam $\mathrm{mol}^{-1} \mathrm{P} 450$ ).

${ }^{\mathrm{c}} \mathrm{Mmol}$ sultam (4b) / mmol total products $(\mathbf{4 b}+\mathbf{4 c})$. 
Table S2. Noncompetitive intermolecular kinetic isotope effect experiments. ${ }^{a}$

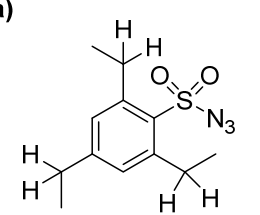

$4 a$

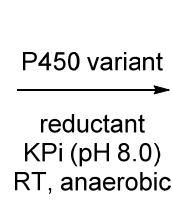
$\mathrm{KPi}(\mathrm{pH} 8.0)$
$\mathrm{RT}$, anaerobic

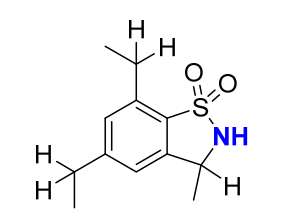

4b

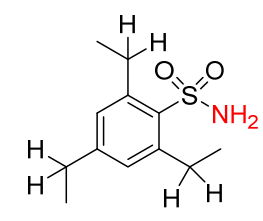

4c b)

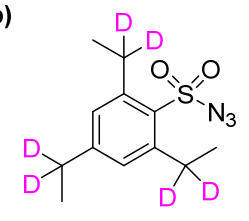

$4 a-d_{6}$

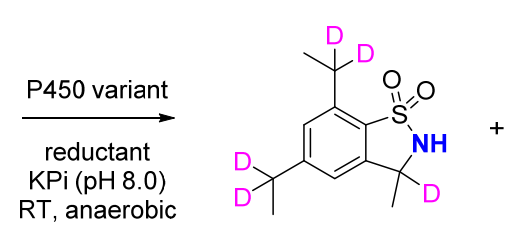

$4 b-d_{5}$

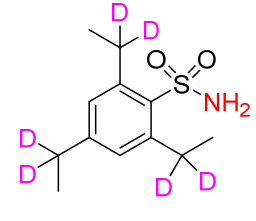

$4 c-d_{6}$

\begin{tabular}{|c|c|c|c|c|c|c|}
\hline \multirow{2}{*}{ Variant } & \multicolumn{3}{|c|}{$4 \mathbf{a}$ to $4 b^{b}$} & \multicolumn{3}{|c|}{$4 a-d_{6}$ to $4 b-d^{b}$} \\
\hline & $\mathrm{K}_{\mathrm{M}}(\mathrm{mM})$ & $\mathrm{k}_{\mathrm{cat}}\left(\times 10^{-3} \mathrm{~s}^{-1}\right)$ & $\mathrm{k}_{\mathrm{cat}} / \mathrm{K}_{\mathrm{M}}\left(\mathrm{M}^{-1} \mathrm{~s}^{-1}\right)$ & $\mathrm{K}_{\mathrm{M}}(\mathrm{mM})$ & $\mathrm{k}_{\text {cat }}\left(\times 10^{-3} \mathrm{~s}^{-1}\right)$ & $\mathrm{k}_{\mathrm{cat}} / \mathrm{K}_{\mathrm{M}}\left(\mathrm{M}^{-1} \mathrm{~s}^{-1}\right)$ \\
\hline FL\#62 & $5.1 \pm 0.3$ & $39 \pm 2$ & $7.6 \pm 0.6$ & $4.3 \pm 0.6$ & $43 \pm 5$ & $10 \pm 1.8$ \\
\hline FL\#62 (H266F,T268V) & $5.2 \pm 0.9$ & $69 \pm 11$ & $13 \pm 3$ & $3.7 \pm 0.2$ & $82 \pm 26$ & $22 \pm 2$ \\
\hline FL\#62 (H266F,T268V,P392G) & $0.41 \pm 0.09$ & $2.8 \pm 0.1$ & $6.8 \pm 1.6$ & $0.35 \pm 0.01$ & $3.0 \pm 0.4$ & $8.6 \pm 1.2$ \\
\hline XplA-RhF & $0.72 \pm 0.03$ & $45 \pm 11$ & $62 \pm 15$ & $0.60 \pm 0.08$ & $44 \pm 2$ & $73 \pm 10$ \\
\hline $\mathrm{BezE}^{\mathrm{c}}$ & $2.7 \pm 0.03$ & $32 \pm 0.7$ & $12 \pm 1.4$ & $6.8 \pm 0.2$ & $138 \pm 39$ & $20 \pm 5.7$ \\
\hline
\end{tabular}

${ }^{a}$ Conditions: $5 \mu \mathrm{M}$ P450, $0.5 \mathrm{mM}$ to $10 \mathrm{mM}$ substrate, $10 \mathrm{mM}$ NADPH as reductant, $50 \mathrm{mM}$ KPi buffer pH 8.0. Reactions were quenched at $10 \mathrm{~min}$ after addition of substrate. ${ }^{b} \mathrm{~K}_{\mathrm{M}}$ and $\mathrm{k}_{\text {cat }}$ values are estimated from Michaelis Menten equation; reported values are mean $\pm \mathrm{SD}^{c} \mathrm{Using}^{10} \mathrm{mM} \mathrm{Na}_{2} \mathrm{~S}_{2} \mathrm{O}_{4}$ as reductant, $50 \mathrm{mM}$ potassium phosphate buffer $\mathrm{pH}=6.0$. 
Table S3. Sequence of the oligonucleotides in 5'-3' direction used for the preparation of engineered cytochrome P450 variants.

\begin{tabular}{|c|c|c|}
\hline Entry & Primer & Sequence (from 5' to 3') \\
\hline 1 & BamHI_2_fwd & GGAAACAGGATCCATCGATGC \\
\hline 2 & SacI_2_rev & AATATCGAGCTCGTAGTTTGTATGATC \\
\hline 3 & BM3RedRev_short & CGGGCTCAGATCTGCTCATG \\
\hline 4 & FL\#62_H266F_for & CTTAATTGCGGGATTTGAAACAAC \\
\hline 5 & FL\#62_H266F_rev & GTTGTTTCAAATCCCGCAATTAAG \\
\hline 6 & FL\#62_E267A_for & GGGACACGCGACAACAAGTG \\
\hline 7 & FL\#62_E267A_rev & CACTTGTTGTCGCGTGTCCC \\
\hline 8 & FL\#62_T268V_for & CGGGACACGAAGTGACAAGTGG \\
\hline 9 & FL\#62_T268V_rev & CCACTTGTCACTTCGTGTCCCG \\
\hline 10 & FL\#62_T438V_for & $\begin{array}{l}\text { GATATTAAAGAAACTTTAGTGTTAAAACCTGA } \\
\text { AG }\end{array}$ \\
\hline 11 & FL\#62_T438V_rev & $\begin{array}{l}\text { CTTCAGGTTTTAACACTAAAGTTTCTTTAATAT } \\
\text { C }\end{array}$ \\
\hline 12 & FL\#62_E267A-T268V_for & GGGACACGCGGTGACAAGTG \\
\hline 13 & FL\#62_E267A-T268V_rev & CACTTGTCACCGCGTGTCCC \\
\hline 14 & FL\#62-P392G-for & GCGTTTAAAGGCTTTGGAAACG \\
\hline 15 & FL\#62-P392G-rev & CGTTTCCAAAGCCTTTAAACGC \\
\hline 16 & FL\#62-F393P-for & GTTTAAACCGCCGGGAAACG \\
\hline 17 & FL\#62-F393P-rev & CGTTTCCCGGCGGTTTAAAC \\
\hline 18 & FL\#62-G394P-for & CCGTTTCCGAACGGTCAGC \\
\hline 19 & FL\#62-G394P-rev & GCTGACCGTTCGGAAACGG \\
\hline 20 & FL\#62-I401P-for & GTGCGTGTCCGGGTCAGC \\
\hline 21 & FL\#62-I401P-rev & GCTGACCCGGACACGCAC \\
\hline 22 & FL\#62-G402P-for & CGTGTATCCCGCAGCAGTTC \\
\hline 23 & FL\#62-G402P-rev & GAACTGCTGCGGGATACACG \\
\hline 24 & XplA_fw & $\begin{array}{l}\text { CAGGTCATATGGGCTCGTCTCATCATCATCATC } \\
\text { ATC }\end{array}$ \\
\hline 25 & XplA_rv & $\begin{array}{l}\text { GGAACCTCGAGTTAGGACAGGACAATCGGCAG } \\
\text { TTTA }\end{array}$ \\
\hline 26 & Xp1A_M322A_fw & $\begin{array}{l}\text { GTTATGGAAGCAATGTTTGAAGCTATGCTGGC } \\
\text { GCAAAGCGCGGAAC }\end{array}$ \\
\hline 27 & XplA_M322A_rv & GCTTCAAACATTGCTTCCATAAC \\
\hline
\end{tabular}




\begin{tabular}{|c|c|c|}
\hline 28 & XplA_Q325A_fw & $\begin{array}{l}\text { ATGGAAGCAATGTTTGAAGCTATGCTGATGGC } \\
\text { GAGCGCGGAACCGG }\end{array}$ \\
\hline 29 & XplA_Q325A_rv & ATAGCTTCAAACATTGCTTCCAT \\
\hline 30 & XplA_V391A_fw & $\begin{array}{l}\text { GCGATTGCCACGATCCTGGTGTTTTATGCAGCG } \\
\text { GGCCACATGGCTA }\end{array}$ \\
\hline 31 & XplA_V391A_rv & AACACCAGGATCGTGGCAATCGC \\
\hline 32 & XplA_M394A_fw & $\begin{array}{l}\text { ACGATCCTGGTGTTTTATGCAGTTGGCCACGCG } \\
\text { GCTATTGGTTACC }\end{array}$ \\
\hline 33 & XplA_M394A_rv & ACTGCATAAAACACCAGGATCGT \\
\hline 34 & XplA_Q438A_fw & $\begin{array}{l}\text { ATCAATGAAATGGTTCGTATGGACCCGCCGGCGCT } \\
\text { GTCGTTTCTGC }\end{array}$ \\
\hline 35 & XplA_Q438A_rv & TCCATACGAACCATTTCATTGAT \\
\hline 36 & XplA_C503S_fw & $\begin{array}{l}\text { AATCTGTCTTTTGGTCTGGGTCCGCACAGCAGCGCC } \\
\text { GGTCAGATTA }\end{array}$ \\
\hline 37 & XplA_C503S_rv & GGACCCAGACCAAAAGACAGATT \\
\hline 38 & bezE_fw & ACACATCATATGGGCGATAGCACCAGCGC \\
\hline 39 & bezE_rv & $\begin{array}{l}\text { ACACATCTCGAGATTCCAGGTAACCGGCAGAT } \\
\text { GAACC }\end{array}$ \\
\hline 40 & bezE_T256V_fw & GTTTGGTGGCTATGAAGTGACCGTTGCG \\
\hline 41 & bezE_T256V_rv & CGCAACGGTCACTTCATAGCCACCAAAC \\
\hline
\end{tabular}




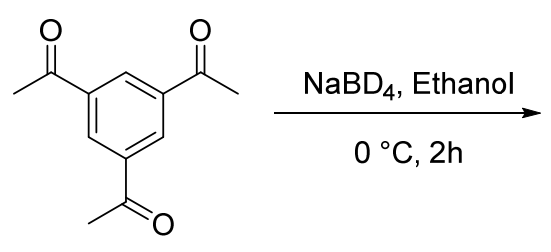

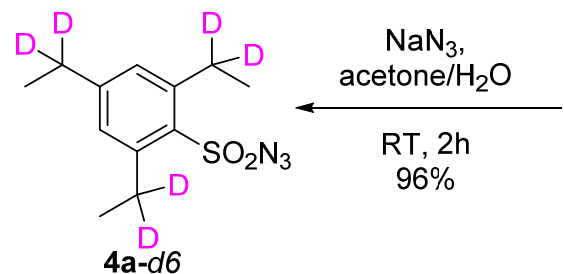<smiles>[R9]C(C)c1cc(C([2H])C)c(S(N)(=O)=O)c(C([2H])C)c1</smiles><smiles>[2H]C(C)(O)c1cc(C([2H])(C)O)cc(C(C)(C)O)c1</smiles>

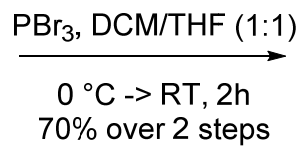<smiles>[2H]C(C)(Br)c1cc(C([2H])(C)Br)cc(C([2H])(C)Br)c1</smiles>
9 LiAID $_{4}$, THF
reflux, 24h
$15 \%$<smiles>[2H]C([2H])([2H])c1cc(C([2H])([2H])C)cc(C([2H])([2H])[2H])c1S(=O)(=O)Cl</smiles><smiles>[2H]C([2H])([2H])c1cc(C([2H])([2H])[2H])cc(C([2H])(C)C)c1</smiles>
$15 \%$

Scheme S1. Synthesis of the deuterated probe molecules 2,4,6-tris(ethyl-1,1-d2)benzenesulfonyl azide $\mathbf{4 a -} d 6$ and 2,4,6-tris(ethyl-1-d)benzenesulfonyl azide $\mathbf{4 a -} d 3$. 


\section{References.}

(1) Sievers, F.; Wilm, A.; Dineen, D.; Gibson, T. J.; Karplus, K.; Li, W.; Lopez, R.; McWilliam, H.; Remmert, M.; Söding, J.; Thompson, J. D.; Higgins, D. G. Fast, Scalable Generation of High-Quality Protein Multiple Sequence Alignments Using Clustal Omega. Mol. Syst. Biol. 2011, 7, 539 .

(2) Brown, N. P.; Leroy, C.; Sander, C. MView: A Web-Compatible Database Search or Multiple Alignment Viewer. Bioinformatics 1998, 14, 380. 